\title{
Expedição Insulina: Avaliando o Acondicionamento da Insulina Durante Atividades de Lazer
}

\author{
Expedition Insulin: Evaluation Storage of Insulin during Leisure Activities
}

\begin{abstract}
Sávio Dias de Paula Mello; ${ }^{\text {a }}$ Lorena Fortuna da Silva ${ }^{\mathrm{a}}$; Ana Beatriz de Andrade Soares de Oliveira ${ }^{\mathrm{a}}$; Fernanda Zerbinato Bispo; Cristiane Rosa Magalhães ${ }^{\text {a; }}$ Júlio Cesar Santos da Silva ; Úrsula Pérsia Paulo dos Santos ${ }^{\text {a }}$, Marcela dos Santos Ferreira*a
\end{abstract}

Centro Federal de Educação Tecnológica Celso Suckow da Fonseca. RJ, Brasil.

*E-mail: cceccella@hotmail.com

\begin{abstract}
Resumo
A má conservação dos frascos e canetas de insulina, decorrentes da exposição a altas temperaturas, é uma das principais causas para um mau controle glicêmico. $\mathrm{O}$ estudo se propôs a realizar algumas pesquisas de campo com o objetivo de avaliar comparativamente as formas de acondicionamento da insulina, em relação a sua temperatura, durante as atividades de lazer. A pesquisa é do tipo descritivo-explicativa, realizada no período de maio de 2018 a abril de 2019. O estudo foi executado áreas de atividades de lazer. Foram realizadas medições de temperatura em frascos e canetas aplicadoras de insulina acondicionadas em recipientes diversos. Algumas formas de acondicionamento não conseguiram manter sempre o frasco da insulina em temperaturas ideais, quando expostas a temperaturas ambientes elevadas. Nas medições dos frascos de insulina, a bolsa térmica vazia ou com somente um gelo flexível, uso de isopor vazio e ausência de recipiente térmico foram medidas ineficazes para a conservação da medicação. Em relação às canetas aplicadoras medições mostraram que só o uso do estojo próprio, recomendado pelo fabricante, não conseguiu manter a insulina em temperatura recomendada. $\mathrm{O}$ conhecimento gerado nesta pesquisa tem o potencial de influenciar positivamente a vida dos usuários de insulina, pois impacta diretamente na forma como se realiza as atividades de lazer. Desta forma, com estas recomendações é possível promover melhor qualidade de vida aos portadores de diabetes mellitus usuários de insulina, já que poderão conciliar seu tratamento medicamentoso com suas atividades de lazer.
\end{abstract}

Palavras-chave: Insulina. Transporte. Temperatura Elevada. Atividades de Lazer.

\begin{abstract}
The bad conservation of insulin vials and pens, due to exposure to high temperatures, is one of the main reasons for poor glycemic control. The study proposed to carry out some field research with the objective of comparatively evaluating the forms of insulin storage, in relation to its temperature, during leisure activities. The research is descriptive-explanatory type, carried out from May 2018 to April 2019. The study was conducted in leisure activities areas. Temperature measurements were made in vials and insulin pens conditioned in different containers. Some storage forms have not always managed to keep the insulin vial at ideal temperatures when exposed to elevated temperatures. In the measurements of insulin vials, the empty thermal bag or with only a flexible ice, the use of empty Styrofoam and the absence of thermal container were ineffective actions for the medication conservation. Regarding the applicator pens, measurements showed that only the use of the case itself, recommended by the manufacturer, failed to keep the insulin at the recommended temperature. The knowledge created in this research has the potential to positively influence the insulin users'lives, as it directly impacts the way leisure activities are performed. Thus, with these recommendations it is possible to improve the quality of life of people with diabetes mellitus, insulin users, once they will be able to reconcile their drug treatment with their leisure activities.
\end{abstract}

Keyword: Insulin. Transportation. Hot Temperature. Leisure Activities.

\section{Introdução}

O Diabetes Mellitus (DM) é uma doença crônica que passou a ser considerado um dos problemas de saúde pública do país. Em 2015, a International Diabetes Federation (IDF) divulgou os países com maiores casos de DM, levando em consideração pessoas entre 20 e 79 anos. Mundialmente estimou-se em 2015 que $8,8 \%$ da população entre 29 e 79 anos (415 milhões de pessoas) vivia com algum tipo de DM e o Brasil assumia a $4^{\circ}$ posição, com 14,3 milhões de casos. Se as tendências atuais persistirem, o quantitativo de pessoas com algum tipo de DM foi projetado para 642 milhões em 2040 (INTERNATIONAL DIABETES FEDERATION, 2018).

O Diabetes Mellitus é caracterizado por uma deficiência parcial ou total da insulina. A doença ocorre quando o pâncreas não é capaz de produzir o hormônio insulina em quantidades suficientes para suprir a demanda do organismo, ou mesmo nem o produz, e também, quando o hormônio não realiza sua função de forma adequada, resultando no aumento da glicemia. Por isso, em alguns casos clínicos há a necessidade da reposição deste hormônio.

Os portadores de DM precisam ser orientados quanto à forma de aplicação da insulina exógena, de natureza igual precisam ser orientados em relação às formas de conservação e transporte do frasco de insulina, pois a conservação correta do frasco de insulina é importante para a manutenção de um bom controle glicêmico, visto que a exposição da insulina a temperaturas elevadas é provavelmente um dos principais razões para resultados insatisfatórios do tratamento e cetoacidose com risco de vida em países com extremas 
condições de clima (PFÜTZNER; PESACH; NAGAR, 2017; MAJALIWA et al., 2010; PRYCE, 2009).

Para a conservação adequada dos frascos de insulina ainda lacrados, os mesmos devem estar a uma temperatura entre 2 ${ }^{\circ} \mathrm{C}$ e $8{ }^{\circ} \mathrm{C}$, não devem ser congelados e caso ocorra, a mesma precisará ser descartada (BRASIL, 2006; SOCIEDADE BRASILEIRA DE DIABETES, 2017).

Durante o transporte da insulina, sendo de curta ou longa distância, os frascos de insulina não devem ser expostos à luz solar, portanto recomenda-se o uso de bolsa térmica ou caixa de isopor, e pode ser mantido em temperatura ambiente entre $15{ }^{\circ} \mathrm{C}$ e $30{ }^{\circ} \mathrm{C}$ (BRASIL, 2006)

Visto a necessidade da boa manutenção das temperaturas da insulina, o projeto de pesquisa denominado Expedição Insulina conduziu um estudo sobre as melhores formas de acondicionamento dos frascos e canetas aplicadoras de insulina especificamente durante atividades de lazer em momentos de alta temperatura ambiente, de forma a mimetizar acontecimentos diários de portadores de DM que se expõem a estas temperaturas. Fundamental salientar, até como meio de enfatizar a importância do estudo que se segue, que a maioria das estatísticas recentes indica que a prevalência de diabetes é mais alta em regiões com alta temperatura e altas incidências são visto em países com períodos frequentes de calor extremo (BARROS; PORTO, 2016). Desta forma, faz-se importante, especialmente para o público brasileiro que se expõem frequentemente a altas temperaturas, estarem informados sobre as diversas formas de armazenamento da insulina com suas respectivas vantagens e desvantagens, e com isso aumentar a promoção à saúde destas pessoas, pois estará reduzindo riscos à saúde sem a necessidade de interrupção de suas atividades diárias de lazer.

Visto que os estudos sobre conservação de insulina em ambiente extradomiciliar não são abundantes, este estudo se propôs a realizar algumas pesquisas de campo com o objetivo de avaliar comparativamente as formas de acondicionamento da insulina, em relação a sua temperatura, durante as atividades de lazer.

\section{Material e Métodos}

Pesquisa do tipo descritivo-explicativa, realizada durante o período de maio de 2018 a abril de 2019 no Estado do Rio de Janeiro. O estudo foi executado, por três pesquisadores, em ambientes extradomiciliares, compondo áreas de atividades de lazer que requerem um período longo fora do domicílio (média de12 horas).

Cada pesquisador munido de frascos de insulina de 10 $\mathrm{ml}\left(\right.$ Humulin $\left.^{\mathrm{R}}\right)$ e canetas aplicadoras de insulina reutilizáveis $\left(\right.$ Humulin $^{\mathrm{R}}$ ) acondicionadas em recipientes diversos, realizaram medições de temperatura ambiente e dos frascos e canetas de insulinas. Para aferição das temperaturas foram utilizados termômetros digitais calibrados com sensor de mínima e máxima $\left(\right.$ INCOTERM $\left.^{\mathrm{R}}\right)$, fixado ao frasco ou caneta aplicadora de insulina, longe do contato direto com o gelo, quando adotado. Para o transporte da insulina foram usados recipientes que acondicionaram os frascos e canetas aplicadoras de insulina, escolhidos de forma a respeitar os mais identificados na literatura (OLIVEIRA et al., 2019), sendo a aplicada a este estudo caixa térmica de EPS de 1,5 litros (Isopor R), bolsa térmica de PVC de tamanho pequeno e médio $\left(\right.$ Nilvan $\left.^{\mathrm{R}}\right)$ com capacidade para cerca de $500 \mathrm{ml}$ e 1 litro, nessa ordem, estojo tipo escolar e estojo térmico próprio para caneta aplicadora de insulina de EPS (Humulin ${ }^{\mathrm{R}}$ ). Também foram empregados, em alguns casos, gelo natural e gelo artificial flexível e rígido $\left(\right.$ gelox $\left.^{\mathrm{R}}\right)$ que eram inseridos no interior dos recipientes em conjunto com a insulina, evitando o contato entre ambos. Todo o material de estudo foi transportado no interior de mochilas de forma a ficarem protegidas de a luz solar diretamente.

Em cada medicação era aferida e registrada a temperatura máxima e mínima tanto do ambiente quanto da insulina. Por dia foram realizadas verificações em 6 frascos e/ou canetas aplicadoras de insulina, sendo que em cada uma destas a temperatura foi verificada 6 a 8 vezes/dia, a depender das mudanças de locais durante o dia, sob a mesma ordem cronológica: na saída do domicílio, no transporte público de ida, local da atividade de lazer, no transporte público de retorno, chegada ao domicílio.

Os locais para as expedições diárias foram escolhidos de forma aleatória encerrando as atividades de lazer e regiões: trilha pelo morro do Pão de Açúcar e ida a Praia Vermelha (bairro da Urca); visita ao AquaRio $^{\mathrm{R}}$ e passeio de barco com destino à Ilha Fiscal promovido pelo Espaço Cultural da Marinha (Centro do Rio de Janeiro); visita a exposições, acrobacias aéreas, shows musicais e demais atividades na comemoração do $145^{\circ}$ aniversário de Santos Dumont organizadas pelo Instituto Histórico-Cultural da Aeronáutica no Museu Aeroespacial (Campo dos Afonsos); participação em exibição do filme Aquaman no cinema do Top Shopping e jogo de boliche no estabelecimento Bowling Show (município de Nova Iguaçu); tour de bicicleta e visita guiada pela Ilha de Paquetá (bairro histórico de Paquetá); mergulho de batismo (município de Arraial do Cabo).

Os valores das temperaturas foram registrados em planilha criada pelos pesquisadores, onde também foi apontada qualquer intercorrência durante as excursões científicas. Em relação aos resultados, estes foram analisados segundo as temperaturas preconizadas pelo Ministério da Saúde (MS) e Sociedade Brasileira de Diabetes (SBD), sendo considerados adequados frascos cuja temperatura não ultrapassa $30{ }^{\circ} \mathrm{C}$ (BRASIL, 2006; SOCIEDADE BRASILEIRA DE DIABETES, 2017).

Os dados foram categorizados por faixas de temperatura $\mathrm{e}$ formas de acondicionamento, sendo calculados, com auxílio do software Excel ${ }^{\mathrm{R}}$ 2010, e apresentados à frequência relativa das medições de temperatura.

Vale ressaltar, que os dados referentes a cada dia de 
pesquisa, foi apresentado em uma mídia social denominado Expedição Insulina, de forma a proporcionar educação em saúde na área de diabetes mellitus.

\section{Resultados e Discussão}

A experiência de avaliar as temperaturas da insulina (frascos ou canetas aplicadoras) ocorreu em diversas formas de acondicionamento e sob exposição de variadas temperaturas ambientes. $\mathrm{O}$ resultado apresentado neste artigo refere-se às medições das temperaturas dos frascos de insulina quando expostos a temperaturas ambientes superiores a $30^{\circ} \mathrm{C}$, pois esta é responsável pela diminuição da atividade da insulina.

Quadro 1 - Frequência relativa das medições das temperaturas dos frascos de insulina quando expostos a temperaturas ambientes acima de $30^{\circ} \mathrm{C}$

\begin{tabular}{|l|c|c|}
\hline Forma de Acondicionamento & $\begin{array}{c}\text { Temperatura } \\
<\mathbf{3 0} \mathbf{}^{\circ}\end{array}$ & $\begin{array}{c}\text { Temperatura } \\
\mathbf{3} \mathbf{3 0} \mathbf{C}\end{array}$ \\
\hline $\begin{array}{l}\text { Frasco de insulina dentro da } \\
\text { bolsa térmica vazia }\end{array}$ & $83,4 \%$ & $16,6 \%$ \\
\hline $\begin{array}{l}\text { Frasco de insulina dentro da } \\
\text { bolsa térmica com dois gelos } \\
\text { flexíveis }\end{array}$ & $100 \%$ & $0 \%$ \\
\hline $\begin{array}{l}\text { Frasco de insulina dentro da } \\
\text { bolsa térmica com um gelo } \\
\text { flexível }\end{array}$ & $79,2 \%$ & $20,8 \%$ \\
\hline $\begin{array}{l}\text { Frasco de insulina dentro da } \\
\text { bolsa térmica com um gelo } \\
\text { rígido }\end{array}$ & $100 \%$ & $0 \%$ \\
\hline $\begin{array}{l}\text { Frasco de insulina dentro da } \\
\text { bolsa térmica com dois gelos } \\
\text { rígidos }\end{array}$ & $100 \%$ & $0 \%$ \\
\hline $\begin{array}{l}\text { Frasco de insulina dentro } \\
\text { da bolsa térmica com gelo } \\
\text { natural }\end{array}$ & 100 & $0 \%$ \\
\hline $\begin{array}{l}\text { Frasco de insulina na caixa de } \\
\text { isopor vazia }\end{array}$ & $83.4 \%$ & $16,6 \%$ \\
\hline $\begin{array}{l}\text { Frasco de insulina na caixa de } \\
\text { isopor com gelo natural }\end{array}$ & $100 \%$ & $0 \%$ \\
\hline $\begin{array}{l}\text { Frasco de insulina na caixa } \\
\text { de isopor com dois gelos } \\
\text { flexíveis }\end{array}$ & $100 \%$ & $0 \%$ \\
\hline $\begin{array}{l}\text { Frasco de Insulina dentro } \\
\text { de frasco plástico com gelo } \\
\text { natural }\end{array}$ & $80 \%$ & $20 \%$ \\
\hline Fonte: Dad dapesqua & \\
\hline
\end{tabular}

Fonte: Dados da pesquisa.

O Quadro 1 ressalta a frequência relativa das medições das temperaturas dos frascos de insulina sob várias formas de acondicionamento quando expostos a temperaturas ambientes impróprias para a conservação da insulina (acima de $30^{\circ} \mathrm{C}$ ). Observa-se, por meio das medições, que algumas formas de acondicionamento não conseguiram manter sempre o frasco da insulina em temperaturas abaixo de $30^{\circ} \mathrm{C}$, sendo estas imprescindíveis para a manutenção de sua viabilidade. Destas, identifica-se bolsa térmica vazia ou com somente um gelo flexível, recipiente de isopor vazio e ausência de recipiente térmico.

Também pode ser observado por meio dos resultados apresentados no Quadro 1 que há diferença das temperaturas dos frascos de insulina quando se altera o modo de induzir a refrigeração. $\mathrm{O}$ uso de apenas um gelo flexível está associado a temperaturas dos frascos de insulina superiores a $30^{\circ} \mathrm{C} \mathrm{em}$
20,8 \% das medições quando acondicionado em bolsa térmica.

No que tange a relação da forma de acondicionamento da caneta aplicadora de insulina com a temperatura da medicação o Quadro 2 mostra que várias medições ultrapassaram as temperaturas recomendadas em períodos que temperatura ambiente esteve acima de $30^{\circ} \mathrm{C}$, inclusive as canetas armazenadas em estojo próprio e recomendado pelo fabricante. No caso das canetas aplicadoras, assim como nos frascos de insulina, pode ser observado que há disparidade das temperaturas das canetas aplicadoras de insulina também está relacionada com a forma de indução da temperatura entre 15 ${ }^{\circ} \mathrm{C}$ a $30{ }^{\circ} \mathrm{C}$. O uso de apenas uma unidade de gelo flexível ou rígido está associado a temperaturas das canetas aplicadoras de insulina desfavoráveis.

Quadro 2 - Relativa das medições das canetas aplicadoras de insulina quando expostas a temperaturas ambientes acima de $30^{\circ} \mathrm{C}$

\begin{tabular}{|l|c|c|}
\hline Forma de Acondicionamento & $\begin{array}{c}\text { Temperatura } \\
<\mathbf{3 0} \mathbf{C}^{\circ}\end{array}$ & $\begin{array}{c}\text { Temperatura } \\
\mathbf{>} \mathbf{3 0} \mathbf{C}\end{array}$ \\
\hline $\begin{array}{l}\text { Caneta de insulina dentro da } \\
\text { bolsa térmica vazia }\end{array}$ & 75 & 25 \\
\hline $\begin{array}{l}\text { Caneta de insulina dentro da } \\
\text { bolsa térmica com dois gelos } \\
\text { flexíveis }\end{array}$ & 100 & 0 \\
\hline $\begin{array}{l}\text { Caneta dentro do estojo tipo } \\
\text { escolar com um gelo rígido }\end{array}$ & 83,4 & 16,6 \\
\hline $\begin{array}{l}\text { Caneta de insulina dentro do } \\
\text { estojo tipo escolar vazio }\end{array}$ & 40 & 60 \\
\hline $\begin{array}{l}\text { Caneta de insulina dentro de } \\
\text { frasco plástico (dentro da bolsa } \\
\text { térmica sem gelo) }\end{array}$ & 83,4 & 16,6 \\
\hline $\begin{array}{l}\text { Caneta de insulina dentro do } \\
\text { estojo próprio do fabricante }\end{array}$ & 85,3 & 14,7 \\
\hline
\end{tabular}

Fonte: Dados da pesquisa.

Outro dado importante obtido a partir da atividade de campo realizado neste estudo é que os frascos de insulina e as canetas aplicadoras tiveram as menores temperaturas aferidas em momentos referentes à saída do domicilio para a atividade de lazer e em ambientes com controle de temperaturas (Ex: metrô). Enquanto as maiores temperaturas verificadas foram em locais abertos sem sombreamento (Ex: passeio de bicicleta) e em horários entre 15:00 h e 16:00 h.

A manutenção da temperatura da insulina é essencial para a estabilidade glicêmica dos portadores de diabetes mellitus, no entanto, a falta de cuidado no armazenamento e transporte fora do ambiente domiciliar em dias de temperatura elevada $\left(>30^{\circ} \mathrm{C}\right)$ pode promover alterações na insulina capazes de interferir em sua eficácia.

Mesmo gradual, a perda de potência da insulina após exposição a elevadas temperaturas, pode introduzir uma variabilidade na dosagem de insulina e os efeitos metabólicos induzidos pela mesma. Existindo casos documentados de insulina que se tornou ineficaz após a exposição ao calor e a luz solar e taxas mais altas de cetoacidose nos que estavam armazenando sua insulina inadequadamente (OLIVEIRA et al., 2019; BRAUNE et al., 2019). Alguns autores discutem que quando um paciente reclama de diferenças no 
efeito metabólico de uma determinada insulina, não deve imediatamente assuma as mudanças nos hábitos do paciente como a causa, mas deve considerar que o paciente está certo em suspeitar que sua insulina possa ser o problema (MIRGHANI et al., 2019; CARTER, 2016).

O presente estudo identificou que alguns frascos e canetas de insulina, mesmo sob algum tipo de acondicionamento, não mantiveram a temperatura recomendada $\left(15^{\circ} \mathrm{C}\right.$ a 30 $\left.{ }^{\circ} \mathrm{C}\right)$, (BRASIL, 2006; SOCIEDADE BRASILEIRA DE DIABETES, 2017). Quando expostos a temperaturas ambientes acima $30^{\circ} \mathrm{C}$. Isso evidencia a importância da orientação aos usuários de insulina sobre uso correto do armazenamento da medicação em dias quentes, especialmente quando dados de pesquisas indicam que um quinto dos entrevistados de sua pesquisa disse que não tomam medidas de proteção até que as temperaturas atinjam valores altos como $38^{\circ} \mathrm{C}$ a $40^{\circ} \mathrm{C}$ (NASSAR; CHILDS; BOYLE, 2010). Extrai-se com isso que essa parcela de usuários que não usam nenhuma forma de acondicionamento, sob temperaturas elevadas, está suscetível a falhas no efeito da insulina.

Em especial, quando se trata do frasco de insulina observou-se que quando a medicação é transportada em recipientes, mesmo denominados térmicos, mas com ausência de gelo (natural, flexível ou rígido) foram detectadas medições de temperaturas acima de $30^{\circ} \mathrm{C}$. Quando analisado o uso dos vários tipos de gelo, foi identificado que a aplicação de uma unidade de gelo flexível não foi suficiente em algumas situações para a manutenção da temperatura durante toda a atividade de lazer. Importante registrar que estudos anteriores demonstram que das pessoas que transportam a insulina para fora do domicilio 33\% dos usuários de insulina a transportam em recipientes térmicos vazios e $31 \%$ usam algum tipo de gelo durante o transporte (NASSAR, CHILDS, BOYLE, 2010). Isto evidencia que existe um quantitativo importante de indivíduos diabéticos que devem ser foco de orientação para o uso de frascos térmicos com a presença de gelo natural, gelo rígido e gelo flexível (mais de uma unidade).

Em relação às canetas de insulina reutilizáveis observouse, que quando é transportada em seu estojo próprio sob temperaturas acima de $30{ }^{\circ} \mathrm{C}$, algumas medições estiveram em temperaturas superiores às recomendadas. A indicação é mantê-la no interior do estojo próprio do dispositivo a uma temperatura ambiente de até $30{ }^{\circ} \mathrm{C}$ (SOCIEDADE BRASILEIRA DE DIABETES, 2017). A partir desta orientação, entende-se que em temperaturas ambientes mais elevadas faz-se necessário algum cuidado extra para a manutenção da temperatura entre $15^{\circ} \mathrm{C}$ a $30^{\circ} \mathrm{C}$.

Ao analisar pesquisas anteriores encontrou-se $34 \%$ dos usuários de caneta aplicadora usando o estojo próprio do fabricante, enquanto $46 \%$ não a guardam dentro do estojo próprio e nem sob outra forma de acondicionamento (OLIVEIRA et al., 2019). Estes dados, associados a atual pesquisa demonstra que existe a necessidade de orientação tanto dos indivíduos usuários do estojo próprio, já que em temperaturas elevadas não consegue manter sua finalidade, como também aos que não usam nenhum recipiente térmico, pois terão mais dificuldade na manutenção de uma temperatura recomendável desta caneta. Pelo presente estudo, o uso de bolsa térmica e algum tipo de gelo fazem-se necessário para a conservação da insulina.

Todavia é importante salientar que tanto o frasco e a caneta de insulina ao serem conservados sob o uso de algum tipo de gelo, não devem manter contato direto com o mesmo, para que não ocorra diminuição da temperatura da medicação (SOCIEDADE BRASILEIRA DE DIABETES, 2017)

Os dados encontrados na pesquisa trazem à tona a importância da educação em saúde aos usuários de insulina, especialmente quando contrastado com dados que evidenciam que em torno de $70 \%$ dos indivíduos portadores de DM disseram ter recebido treinamento sobre a conservação da insulina (OLIVEIRA et al., 2019; NASSAR; CHILDS; BOYLE, 2010; KOCH et al., 2019; MOREIRA et al., 2018). No entanto apenas $41 \%$ de todos os entrevistados afirmaram que não receberam informações sobre os efeitos do calor sob a insulina e 71\% limitaram a exposição ao calor a menos de uma hora transporte(NASSAR; CHILDS; BOYLE, 2010). E que os jovens relatam terem momentos de lazer impedidos pelo medo de danificar a insulina (VARGAS et al., 2019; WEIL, 2002). De forma a reduzir este déficit de informação, os portadores de DM deveriam receber orientações sobre os melhores métodos de conservação, assim como, informação sobre as temperaturas mínimas e máximas a que o medicamento pode ser submetido, sobre a validade do medicamento quando em uso e os locais mais recomendados para o armazenamento, já que estes são os erros mais comuns desta população (GAERTNER et al., 2015; SOUSA; VASCONCELOS, 2014; PEREIRA et al., 2016; ARRAIS; OLIVEIRA, 2016). Por isso, é imprescindível enfatizar na educação em saúde as diversas possibilidades de conservação da insulina em dias de temperatura elevada. Esses resultados indicam que o aumento da sensibilização pública é um tópico necessário e que o gerenciamento de diabetes mellitus no calor precisa ser abordado especificamente nas diretrizes atuais de tratamento (AMERICAN DIABETES ASSOCIATION, 2016). E desta forma, sendo possível manter atividades de lazer por estes indivíduos.

Algumas limitações podem ser descritas perante este estudo e que ao mesmo tempo devem ser um estímulo para futuras pesquisas neste campo. Uma destas é a existência de pouca informação disponível ao público sobre o impacto na potência da insulina quando ocorrem elevações de temperatura, além do número de publicações recentes sobre esta temática ainda é escassa (BRAUNE et al., 2019).

\section{Conclusão}

Após as medições de temperatura dos frascos e canetas aplicadoras de insulina, armazenadas em diversos tipos de recipientes, observou-se que algumas formas de 
acondicionamento, assim como o tipo de gelo usado, não foram suficientes para a manutenção da temperatura da insulina abaixo de $30^{\circ} \mathrm{C}$.

Em relação ao frasco de insulina, recomenda-se sempre o suo de recipiente térmico, como isopor ou bolsa térmica, tendo em seu interior gelo natural ou duas unidades de gelo flexível ou rígido. Importante salientar a necessidade do cuidado deste gelo não entrar em contato direto com a insulina.

Já em relação às canetas aplicadoras de insulina reutilizáveis, recomenda-se sob temperaturas elevadas, o transporte em recipientes térmicos contendo gelo natural ou duas unidades de gelo flexível ou rígido, já que o fabricante da insulina só recomenda seu uso em temperatura ambiente abaixo de $30^{\circ} \mathrm{C}$. O estudo também conseguiu demonstrar que estes estojos não conseguem manter sempre a temperatura da insulina em condições ideais. Assim como, nos frascos de insulina, deve-se ter atenção a não manter a caneta aplicadora em contato direto com o gelo, pois pode danificar o sistema mecânico da mesma.

Todo conhecimento gerado nesta pesquisa tem o potencial de influenciar positivamente a vida dos usuários de insulina, pois impacta diretamente na forma como se realiza as atividades de lazer. Desta forma, com estas recomendações é possível promover melhor qualidade de vida aos portadores de diabetes mellitus usuários de insulina, já que poderão conciliar seu tratamento medicamentoso com suas atividades de lazer.

\section{Referências}

AMERICAN DIABETES ASSOCIATION. Standards of medical care in diabetes care. Diabetes Care, v.22, n.1, p.1 -119, 2016. doi: $10.2337 / \mathrm{dc} 10-\mathrm{S} 011$

ARRAIS, E.C.; OLIVEIRA, B.F.S. Acondicionamento da insulina no domicílio por diabéticos. Revista Recien, v.6, n.16, p.21-31, 2016. doi:10.24276/rrecien2358-3088.2016.6.16.21-31

BARROS, J.M.S.; PORTO, M.L.S. Perfil dos usuários diabéticos da unidade básica de saúde do município de Santana do Seridó - RN. Temas em Saúde, v. 16, n. 2, p.226-238, 2016. doi: $10.29327 / 213319$

BRASIL. Ministério da Saúde. Secretaria de Atenção à Saúde. Departamento de Atenção Básica. Cadernos de Atenção Básica: Diabetes Mellitus. Brasília: MS, 2006.

BRAUNE, K. et al. Storage conditions of insulin in domestic refrigerators and carried by patients - insulin is often stored outside recommended temperature range. Diabetes, v.67, n.1, p.141-160, 2018. doi: 10.2337/db18-141-lb

CARTER, A.W.; HEINEMANN, L.; KLONOFF, D.C. Quality Control of Insulins and Biosimilar Insulins. $J$.
Diabetes Scie. Technol., v.10, n.4, p.811-815, 2016. doi: 10.1177/1932296816638923

GAERTNER, F. et al. Procedimentos relacionados ao uso de insulina por portadores de diabetes mellitus tipo I e tipo II. Rev. Contexto Saúde, v. 14, n. 27, p. 44-53, 2015. doi: 10.21527/21767114.2014.27.44-53

INTERNATIONAL DIABETES FEDERATION. IDF Diabetes Atlas, 8th edition. 2018.

$\mathrm{KOCH}, \mathrm{M}$. et al. Avaliação sobre o armazenamento da insulina em uma amostragem de usuários. Rev. UNINGÁ, v. 56, n. 1, p. 17-25, 2019.

MAJALIWA, E.S. et al. Management of diabetic ketoacidosis in children and adolescents in sub-saharan Africa: a review. East Afr. Med. J., v.87, p.167-173, 2010. doi: 10.4314/eamj.v87i4.62207

Mirghani H.O. et al. The site of insulin storage inside domestic refrigerators is associated with ketoacidosis and hypoglycemic episodes among patient with diabetes mellitus, in Tabuk City, The Kingdom of Saudi Arabia. AMJ, v.12 n.12, p.350-355, 2019. doi: 10.35841/1836-1935.12.12.350-355

MOREIRA, T.R. et al. Fatores relacionados à autoaplicação de insulina em indivíduos com diabetes mellitus. Rev. Gaúcha Enferm., v.39, p.1-9, 2018. doi: 10.1590/1983-1447.2018.2017006

NASSAR, A.A. et al. Diabetes in the Desert: What Do Patients Know about the Heat?. J. Diabetes Scie. Technol., v. 4, n. 5, p.1156-1163, 2010. doi:10.1177/193229681000400514

OLIVEIRA, A.B.A.S. et al. Conhecimento de portadores de diabetes mellitus acerca da conservação da insulina. Saúde (Santa Maria), v.45, n.2, p.1-10, 2019. doi: 10.5902/2236583437342

PEREIRA, F.G. et al. Fatores relacionados a utilização de insulina em diabéticos acompanhados pela estratégia saúde da família. Rev. APS, v. 19, n. 1, p.58-66, 2016.

PFÜTZNER, A.; PESACH, G.; NAGAR, R. Technologyderived storage solutions for stabilizing insulin in extreme weather conditions I: the ViViCap-1 device. Expert Opinion on Drug Delivery, v.14, n.6, p.709-714, 2017. doi: 10.1080/17425247.2017.1316261

PRYCE, R. Diabetic ketoacidosis caused by exposure of insulin pump to heat and sunlight. Bmj, v.338, n.51, p.338-2218, 2009. doi:110.1136/bmj.a2218

SOCIEDADE BRASILEIRA DE DIABETES (SBD). Diretrizes sociedade brasileira de diabetes 2017-2018. Rio de Janeiro: Clannad, 2017.

SOUZA, H.K.O.; VASCONCELOS, R.B. Perfil dos usuários de insulina atendidos em uma unidade de saúde. REVISA, v. 2, p. 141-152, 2014.

VARGAS, D.M. et al. O uso da caneta injetora de insulina no cotidiano: percepções do adolescente. Ciênc. Saúde, v. 12, n. 3, p. 33426-33432, 2019. doi:10.15448/1983-652x.2019.3.33426

WEIL, R.M. Sports \& fitness: planning an active summer. Diabetes Self Managed, v. 9, n.3, p. 89-94, 2002. 